\title{
LES 'PERSÉCUTIONS LIÉES AU GENRE' EN SUISSE : LES FRONTIÈRES DU DROIT D'ASILE EN QUESTION
}

\author{
Jonathan Miaz
}

L'Harmattan | Cahiers du Genre

\author{
$2014 / 2-n^{\circ} 57$ \\ pages 55 à 75
}

\section{ISSN 1298-6046}

Article disponible en ligne à l'adresse:

http://www.cairn.info/revue-cahiers-du-genre-2014-2-page-55.htm

Pour citer cet article :

Miaz Jonathan, «Les 'persécutions liées au genre' en Suisse : les frontières du droit d'asile en question », Cahiers du Genre, 2014/2 n 57, p. 55-75.

Distribution électronique Cairn.info pour L'Harmattan.

(c) L'Harmattan. Tous droits réservés pour tous pays.

La reproduction ou représentation de cet article, notamment par photocopie, n'est autorisée que dans les limites des conditions générales d'utilisation du site ou, le cas échéant, des conditions générales de la licence souscrite par votre établissement. Toute autre reproduction ou représentation, en tout ou partie, sous quelque forme et de quelque manière que ce soit, est interdite sauf accord préalable et écrit de l'éditeur, en dehors des cas prévus par la législation en vigueur en France. II est précisé que son stockage dans une base de données est également interdit. 


\title{
Les 'persécutions liées au genre' en Suisse : les frontières du droit d'asile en question
}

\author{
Jonathan Miaz
}

\begin{abstract}
Résumé
À travers le cas suisse, cet article analyse l'intégration d'une dimension de genre dans la définition juridique de la notion de réfugié•e. L'auteur y montre comment différents niveaux de la chaîne du droit ont contribué à cette évolution, laquelle est donc appréhendée à la fois par le haut (instances internationales, débats parlementaires, jurisprudence), mais aussi par le bas, dans les pratiques internes de l'administration. En mettant en lien ces différents niveaux, l'auteur insiste sur le rôle important que jouent les fonctionnaires dans la production des normes juridiques (et non pas seulement dans leur seule application). Enfin, il montre que, si les 'persécutions liées au genre' sont davantage prises en compte, elles restent des motifs ‘de seconde zone’ dont le caractère politique demeure dénié. ${ }^{1}$
\end{abstract}

SUISSE — DROIT D'ASILE — RÉFUGIÉ·E·S—PERSÉCUTIONS LIÉES AU GENRE

Depuis les années 1980, des critiques féministes ont dénoncé le caractère androcentré de la définition juridique $d u$ réfugié (Gafner, Schmidlin 2007 ; Freedman 2008, 2010a, 2010b ; Barzé 2012) en insistant sur la nécessité de prendre en compte la dimension genrée des persécutions. Selon ces travaux, cette définition reposerait sur des expériences principalement masculines. En

${ }^{1}$ Je remercie Fiona Friedli et Bernard Voutat pour leur relecture attentive, ainsi que les lecteurs et lectrices anonymes et les coordinatrices du numéro pour leurs commentaires. 
ignorant le genre, le sexe ou encore l'orientation sexuelle comme causes de persécutions, elle ne tiendrait pas compte de la manière différenciée dont les femmes sont affectées par les conflits, les guerres ou encore les violations des droits humains, et ne reconnaîtrait pas certaines pratiques menées par des femmes comme politiques au même sens et au même titre que pour les hommes. Plus généralement, elle n’intégrerait pas les persécutions visant spécifiquement les femmes. Comme le souligne Magalie Gafner (2003) :

Une opposition aux règles de comportement imposées spécifiquement aux femmes n'est pas considérée comme une opposition politique.

Progressivement, cependant, les instances internationales ont intégré cette critique dans des lignes directrices à l'intention des États. En Suisse, différents acteurs se sont fait l'écho de ces débats. Depuis 1998, la loi suisse sur l'asile établit qu'il « y a lieu de tenir compte des motifs de fuite spécifiques aux femmes" (art. 3 al. 2 LAsi). Au sein de l'Office fédéral des migrations (ODM) - administration chargée d'instruire les demandes d'asile —, ces évolutions se sont traduites par l'élaboration d'une pratique interne en matière de 'persécutions liées au genre' (Geschlechtsspezifische Verfolgung, dite pratique Gespever), ce terme de 'pratique' désignant un dispositif d'interprétation des textes juridiques destiné à orienter leur application à des situations déterminées.

Différents travaux ont insisté sur l'importance du genre ${ }^{2}$ - ses implicites et ses effets - dans notre compréhension de l'action publique (Jenson, Lépinard 2009) et du droit (Lépinard 2006 ; Bereni et al. 2009, 2010), non seulement à propos de politiques concernant directement l'égalité des sexes, mais aussi, vu sa dimension transversale, dans des domaines où le genre n'en est pas l'objet explicite (Bereni et al. 2012). Ainsi en va-til du droit d'asile où cette dimension a été étudiée, notamment,

\footnotetext{
2 À propos de la notion de genre, il convient de distinguer sa définition en tant que concept scientifique désignant un rapport social diviseur (Bereni et al. 2012, p. 10), de celle retenue par l'administration en termes d' " appartenance sexuelle », d' « orientation sexuelle » et d' « identité de genre ». La notion de " persécutions liées au genre » est considérée ici comme une catégorie d’action publique.
} 
en relation avec les parcours migratoires, la place des femmes dans les procédures suivies par les autorités, les 'persécutions liées au genre' (Bhabha 1996 ; Bohmer, Shuman 2008 ; Freedman 2008) ou encore, plus spécifiquement, l'orientation sexuelle des demandeurs et demandeuses d'asile (Morgan 2006 ; Kobelinsky 2012). Suivant la formule d'Éléonore Lépinard (2006, p. 47), il importe d'entrer dans la «boîte noire de la production de la norme juridique »: parce que le droit, en tant qu'expression formalisée d'une matrice cognitive, participe de la construction sociale de la réalité, il contribue, en l’occurrence, à définir la notion de réfugiée et donc à circonscrire le cercle des personnes susceptibles d'entrer dans le cadre d'une 'politique d'asile'.

En Suisse, l'incorporation du genre dans le droit d'asile intervient progressivement à plusieurs niveaux, dans le cadre de ce que Pierre Lascoumes (1990) appelle « un processus de création normative continu ». Elle se réalise en effet aussi bien par le haut, via les instances internationales, les débats parlementaires et certaines évolutions jurisprudentielles, que par le bas, à l'interne de l'administration, dont il faut considérer le rôle important dans la production (et non pas dans la seule application) des normes juridiques (Chevallier 2011). À partir d'une recherche portant sur les usages sociaux du droit en matière d'asile en Suisse ${ }^{3}$, cet article propose une analyse des modalités par lesquelles les différents niveaux de la chaîne du droit (révisions législatives, changements réglementaires et jurisprudentiels, modifications des 'règles secondaires d'application') ont contribué à faire évoluer la définition juridique de la notion de réfugié•e en y intégrant une dimension de genre.

\footnotetext{
${ }^{3}$ Cet article s'appuie sur une enquête ethnographique menée entre 2010 et 2013 dans le cadre d'une thèse de doctorat en science politique. J'ai procédé à plusieurs stages d'observation (Centre d'enregistrement et de procédure et Centre administratif de l'ODM ; bureaux de consultation juridique) et à des entretiens ( $n=95)$ avec des fonctionnaires, des juristes, avocat·e·s et bénévoles de services d'aide juridique ainsi qu'avec des juges, des greffiers et des greffières du Tribunal administratif fédéral (TAF). Ces données sont complétées par une importante recherche documentaire (archives fédérales en ligne, rapports, dossiers de presse, jurisprudence, textes juridiques).
} 


\section{Des 'motifs de fuite spécifiques aux femmes' reconnus dans la loi}

Entrée en vigueur en 1981, la première loi sur l'asile (LAsi), en dépit des nombreuses révisions dont elle a été l'objet, n’a pas modifié la définition de la notion de réfugié·e, reprise pour l'essentiel de la Convention de Genève de 1951 et du Protocole de New-York de 1967. Le premier alinéa de l'article 3 LAsi prévoit que :

Sont des réfugiés les personnes qui, dans leur État d'origine ou dans le pays de leur dernière résidence, sont exposées à de sérieux préjudices ou craignent à juste titre de l'être en raison de leur race, de leur religion, de leur nationalité, de leur appartenance à un groupe social déterminé ou de leurs opinions politiques.

Le deuxième alinéa précise que :

Sont notamment considérées comme de sérieux préjudices la mise en danger de la vie, de l'intégrité corporelle ou de la liberté, de même que les mesures qui entraînent une pression psychique insupportable.

Les causes de persécution ${ }^{4}$ renvoient à des appartenances diverses, énumérées de façon limitative dans la loi, même si la mention générale de "l'appartenance à un groupe social déterminé » pourrait être de nature à conférer une certaine ouverture à cette définition. En tous les cas, le genre, le sexe ou l'orientation sexuelle n'apparaissent pas explicitement dans les causes de persécution figurant au premier alinéa. C'est en 1998, à la faveur d'une révision d'ensemble de la LAsi, que cette définition de la notion de réfugié est amendée. Le premier alinéa n'est pas modifié, mais le second précise, in fine, qu' « il y a lieu de tenir compte des motifs de fuite spécifiques aux femmes". Autrement dit, l'intégration dans la loi d'une problématique de genre intervient non au niveau de la définition même du réfugié figurant au premier alinéa, qui détermine les raisons de cer-

\footnotetext{
${ }^{4}$ Le terme de persécution fait l'objet de différentes interprétations juridiques et administratives. L'art. 3 LAsi ne le mentionne d'ailleurs pas expressément et y substitue la notion de "sérieux préjudice ». Cf. Manuel de procédure de l’ODM (2008), « Chapitre D $\S 1$. La notion de réfugié », disponible sur le site de l'ODM : www.bfm.admin.ch
} 
taines persécutions pouvant conduire à un statut de réfugié, mais par le biais d'une précision insérée dans le second alinéa, qui porte sur la notion de persécution, à laquelle sont finalement rattachés des motifs de fuite spécifiques aux femmes.

\section{Des instances internationales aux relais parlementaires}

La problématique des 'persécutions liées au genre' a été progressivement prise en compte par la communauté internationale à partir des années 1980. Le Haut commissariat des Nations unies pour les réfugiés (HCR) - reprenant alors les mesures préconisées par la $4^{\mathrm{e}}$ Conférence mondiale sur les femmes tenue à Pékin en 1995 - va contribuer à ce que s'élaborent divers instruments visant à prendre en considération les expériences spécifiques des migrantes dans les procédures d'asile nationales. Dans ses lignes directrices sur la protection des femmes réfugiées de 1991, le HCR invite les États à admettre que les requérantes d'asile menacées pour avoir transgressé le code moral de la société dans laquelle elles vivent sont le fait de l'appartenance à un 'groupe social déterminé'. Des directives ultérieures exigent ensuite des États qu'ils prennent des mesures pour prendre en compte la spécificité des persécutions subies par les femmes et pour sensibiliser à la spécificité de certaines problématiques 'culturelles' liées au sexe ${ }^{5}$ les personnes chargées de se prononcer sur les demandes d'asile. De même, les instances européennes ont élaboré des lignes directrices dans ce sens. Enfin, c'est dans les années 2000 que les persécutions en raison de l'orientation sexuelle et de l'identité de genre ont progressivement été prises en compte par les instances internationales en tant que 'persécutions liées au genre'. Toutefois, comme le souligne

\footnotetext{
${ }^{5}$ Différents documents du HCR et de la Conférence mondiale sur les femmes à Pékin sont cités dans un rapport de l’ODM (2005), « La situation des femmes dans la politique d'asile - appréciation des aspects spécifiques aux femmes et liés au sexe en procédure d'asile ». Rendu en août 2005, ce rapport est rédigé en réponse à une intervention parlementaire (postulat Menétrey-Savary : réf. 00.3659). Dans ce postulat, la conseillère nationale Anne-Catherine MenétreySavary (Les Verts) demande que le Conseil fédéral présente un rapport sur la situation des femmes dans la politique d'asile de la Suisse, afin d'établir si l'interprétation de la disposition figurant à l'article 3 al. 2 LAsi permet de garantir l'accueil et la protection des femmes confrontées à des 'persécutions liées au genre'.
} 
Jane Freedman (2008), si ces normes sont acceptées au niveau international, le processus d'adoption et de mise en œuvre au niveau national ne va pas de soi et dépend de leur appropriation par des acteurs étatiques et associatifs.

En Suisse, la révision de la loi, en 1998, fait suite à plusieurs interventions parlementaires qui ont relayé dans l'arène politique les mobilisations féministes et au débat sur la scène internationale autour de la question des persécutions spécifiques aux femmes. À l'instar des motions Gurtner (Parti suisse du travail) en 1985 et 1987, elles demandent tantôt des informations sur la situation des femmes réfugiées en Suisse, tantôt un examen différencié des demandes d'asile selon l'appartenance sexuelle ou la reconnaissance de motifs spécifiques d'octroi de l'asile (viols, mutilations génitales, violences sexuelles). Des interventions parlementaires abordent aussi plus directement les persécutions subies par les femmes dans certains conflits (guerre en exYougoslavie, guerre civile en Algérie). En 1992, cette problématique est abordée dans un rapport du Bureau fédéral de l'égalité entre femmes et hommes (Hausammann 1992). Sur le plan judiciaire, on trouve peu d'arrêts sur les 'persécutions liées au genre' dans la jurisprudence de la Commission suisse de recours en matière d'asile (CRA) durant les années $1990{ }^{6}$. De son côté, l'administration en charge d'instruire les demandes d'asile — l'ODR puis l'ODM depuis $2005^{7}$ — a progressivement mis en place des formations destinées à son personnel. Dès 1996, une circulaire est adoptée, qui contient un catalogue de mesures concernant les procédures d'instruction des demandes d'asile, prévoyant notamment des modalités spécifiques d'audition des personnes invoquant des causes de persécutions liées au genre, comme, par exemple, l'audition par une personne du

\footnotetext{
${ }^{6}$ On peut mentionner JICRA 1993/9 consid. 5c sur la crainte de viols, d'enlèvements et de mariages forcés par les chrétiens syro-orthodoxes en Turquie qui peut être déterminante en matière d'asile ; JICRA 1994/5 sur la persécution réfléchie ; ou JICRA 1996/17 sur la question d'un viol commis par des policiers et les problèmes touchant à la " pertinence et à la crédibilité d'un tel acte en matière d'asile ». En 2007, la juridiction en matière d'asile a été transférée au Tribunal administratif fédéral (TAF), au moment de sa mise en place.

${ }^{7}$ L’Office fédéral des migrations (ODM) est créé en 2005 suite à la fusion entre l'Office fédéral des réfugiés (ODR) et l'Office fédéral de l'immigration, de l'intégration et de l'émigration (IMES).
} 
même sexe (Barzé 2012). Ces mesures comprennent aussi la récolte d'informations approfondies concernant les pays de provenance sur la situation juridique et sociale des femmes, ainsi que la mise en commun des décisions afin d'assurer une pratique uniforme ${ }^{8}$.

La révision de l'article 3 LAsi n’a pas modifié la définition de la notion même de réfugié, se limitant à prévoir une clause concernant les motifs de fuite. Dans son Message du 4 décembre $1995^{9}$, le Conseil fédéral justifie la non-extension de la notion de réfugié aux victimes de "persécutions fondées sur le sexe » par le fait que la Suisse s'écarterait de la définition figurant à l'article 1A de la Convention de Genève. Mais il évoque aussi d'autres raisons, notamment « un effet d'attraction indésirable » qu'une modification matérielle de la notion de réfugié pourrait provoquer. Selon le gouvernement, «les limites des persécutions fondées sur le sexe sont peu claires " et un élargissement de la définition juridique du réfugié " reviendrait à lancer un signal international ». La question des 'abus', centrale et récurrente dans les discours politiques et médiatiques à propos des requérants d'asile, apparaît en creux, d'autant plus quand le Conseil fédéral poursuit son argumentation dans ces termes :

Une persécution fondée sur le sexe serait d'autant plus facile à motiver qu'il n'existerait pas de possibilité d'exiger la preuve d'activités religieuses ou politiques antérieures. En règle générale, il suffirait qu'une femme explique de manière motivée que, pour avoir mis en pratique les normes sociales valables en Suisse, elle risque des persécutions dans son pays (ibid.).

Alors que de nombreuses associations de femmes et de défense du droit d'asile demandaient d'ajouter le sexe aux autres causes de persécution ${ }^{10}$, les autorités n’ont pas jugé nécessaire de modifier la disposition légale sur ce point. La solution finalement retenue, qui n’élargit pas directement la notion de réfugié,

\footnotetext{
${ }^{8}$ ODM (2005), « La situation des femmes dans la politique d'asile », opus cit.

${ }^{9}$ Message du Conseil fédéral concernant la révision totale de la loi sur l'asile du 4 décembre 1995, FF 1996 II 40ss.

${ }^{10}$ Selon le Postulat Menétrey-Savary (00.3659).
} 
est alors présentée comme une forme de «compromis " ${ }^{11}$. Selon les autorités, bien que les persécutions spécifiques au sexe ne soient pas reconnues comme motifs d'asile à part entière, l'ajout figurant au second alinéa de la disposition pourra inciter l'ODM à développer une 'pratique' en la matière, celle que l'office fédéral désigne sous la dénomination de 'persécutions liées au genre’ : pratique Gespever. Dans la continuité de la révision de la LAsi, le Conseil fédéral adopte une réglementation d'application (OA1) qui intègre les directives antérieures de l'administration, en prévoyant, notamment, une audition par "des personnes du même sexe " en cas d'indices de persécutions « de nature sexuelle ».

Dans les années 2000, la question des 'persécutions liées au genre' continue à se poser dans l'arène politique. Au niveau de l'administration, elle se traduit, en outre, par la mise en place de la pratique Gespever et donne lieu à diverses jurisprudences. Depuis 1998, près d'une dizaine d'interventions parlementaires ont abordé directement ou indirectement la question des 'persécutions liées au genre'. En 2005, dans un rapport qui répond au Postulat Menétrey-Savary, l'ODM considère avoir pris en compte la situation des femmes dans la procédure d'asile en adoptant un certain nombre de mesures, et en insistant sur l'importance d'un traitement différencié des demandes d'asile en fonction des spécificités liées au sexe :

La mention expresse des motifs "spécifiques aux femmes 》 doit faciliter une prise de conscience face à la profonde gravité de certains préjudices que les hommes ne connaissent à peine voire pas du tout (ibid.).

\section{Évolution des termes et des motifs de fuite reconnus}

Au niveau international et à l'interne de l'ODM, la terminologie utilisée a évolué. Alors que dans les années 1990 et au début des années 2000, on parlait de 'persécutions spécifiques aux femmes', de 'persécutions liées au sexe' ou de 'persécutions sexo-spécifiques', le terme de 'persécutions liées au genre' ${ }^{12}$ semble s'être imposé au niveau international et dans la pratique

\footnotetext{
${ }^{11}$ ODM (2005), opus cit.

${ }^{12}$ En allemand, le terme Geschlecht désigne indistinctement 'sexe' et 'genre'.
} 
interne développée par l’ODM (Barzé 2012). On passe ainsi d'une perspective centrée sur les femmes à une perspective de genre qui permet d'élargir la notion de 'groupe social déterminé' aux minorités sexuelles victimes de persécutions ${ }^{13}$. Si le manuel de procédure d'asile de l'ODM mentionne encore, en 2008, les 'persécutions liées au sexe', l'usage de la notion de genre se généralise à l'interne de l'ODM, notamment à travers des formations, et suit les recommandations formulées par les instances internationales ainsi que par certaines ONGs de prendre en compte les motifs de persécution liés à l'orientation sexuelle et à l'identité de genre ${ }^{14}$.

Les années 2000 sont également marquées par les revendications de mobilisations LGBT $^{15}$ pour la reconnaissance des persécutions en raison de l'orientation sexuelle et de l'identité de genre. Cette question est portée dans l'arène parlementaire, notamment en 2009, à travers le dépôt d'une motion de la députée Prelicz-Huber (Les Verts). Elle se fonde sur une étude de Martin Bertschi (2007) selon laquelle, entre 1993 et 2007, seuls 4 dossiers sur les 90 consultés où étaient invoqués des motifs liés à l'orientation sexuelle, ont été reconnus comme donnant droit à la qualité de réfugié. La députée propose que ces motifs figurent expressément au second alinéa de l'art. 3 LAsi. Suivant l'avis du Conseil fédéral, le parlement s'est

\footnotetext{
${ }^{13}$ Dans ce sens, on peut citer la recommandation 1470 (2000) de l'Assemblée parlementaire du Conseil de l'Europe, les principes directeurs du HCR (2002 et 2012) ainsi que les Principes de Jogjakarta de 2007 qui invitent à reconnaître les persécutions liées à l'orientation sexuelle et à l'identité de genre comme des motifs d'asile en utilisant le motif de persécution liée à l'appartenance à un certain groupe social.

${ }^{14}$ On peut relier cette évolution à la stratégie de gender mainstreaming adoptée, entre autres, par le HCR dans la continuité de la Conférence de Pékin de 1995. Cette stratégie vise à intégrer une perspective d'égalité de genre dans ses activités (politiques publiques, programmes). Le gender mainstreaming, sa mise en œuvre et sa définition font toutefois l'objet de critiques (voir notamment Freedman 2010b).

${ }^{15}$ L'acronyme LGBT (Lesbiennes, Gays, Bi, Trans) peut être complété pour inclure les personnes intersexuées ou queer (LGBTIQ). Les responsables de la pratique de l'ODM mentionnent en entretien cette évolution (LGBTIQ), mais elles utilisent surtout l'acronyme LGBT. Par la suite, l'acronyme SOGI (Sexual Orientation and Gender Identity) adopté par le HCR dans ses directives de 2012 a également été retenu par l’ODM à la place de LGBT.
} 
opposé à cette modification de la loi, ne l'estimant pas nécessaire du fait que l'ODM tiendrait déjà compte des persécutions liées à l'orientation sexuelle, en les rattachant à l'un des motifs d'asile énoncés dans la loi, à savoir «l'appartenance à un groupe social déterminé ».

On le voit, les dispositions légales et réglementaires sur les 'persécutions liées au genre' ont évolué à travers la révision de 1998, sans toutefois affecter le cœur de la définition du réfugié, ce que certaines critiques ont d'ailleurs relevé. Ainsi, s'il a progressivement été possible d'accorder l'asile à des personnes victimes de violences sexuelles et si les persécutions spécifiques aux femmes sont prises en compte dans les textes juridiques et dans la pratique de l'office, il n'est dit nulle part que le genre, le sexe ou la sexualité constituent des motifs d'asile (au même titre que la race, la religion, la nationalité, ou les opinions politiques).

Sur le plan jurisprudentiel, il faut encore signaler deux décisions très importantes rendues en 2006 par la Commission suisse de recours en matière d'Asile (CRA) et qui vont influencer considérablement la prise en compte des 'persécutions liées au genre' au niveau de l'administration. Dans un premier arrêt (JICRA 2006/32), la Commission a reconnu le 'rapt nuptial' comme motif d'asile et a jugé qu'il fallait offrir une protection aux femmes victimes de telles pratiques lorsque les États ne parviennent pas à interdire ou empêcher certaines 'traditions communautaires'. En lien avec cette décision, la Commission a également rendu un second arrêt dont la portée est plus générale (JICRA 2006/18), en ce qu'il sanctionne un changement fondamental de jurisprudence. Cet arrêt marque le passage de la théorie dite de l' 'imputabilité' à celle dite de la 'protection'. Selon la première approche, pour que la qualité de réfugié soit reconnue, il fallait que l’État soit responsable de la persécution ou qu'il y ait une absence de volonté de protection de sa part face à des "persécutions de tiers » (Barzé 2012). En revanche, selon la théorie de la protection, la question juridique à trancher n'est plus de savoir si les autorités étatiques peuvent être tenues pour responsables directement ou indirectement des persécutions subies, mais de savoir si la personne peut obtenir ou non une protection dans son pays d'origine. Dans cette perspective, la qualité de réfugié peut être reconnue dans le cas de persécutions 
commises par des personnes privées lorsque l'État d'origine est incapable de protéger les personnes. On saisit l'importance de cette jurisprudence s'agissant de certaines 'persécutions liées au genre' qui sont le fait de tiers (mutilations génitales féminines, mariage forcé, répression des homosexuel-le·s, crimes d'honneur, violences domestiques, etc.).

\section{Quand les frontières du droit d'asile bougent par le bas}

Si les frontières du droit d'asile se redéfinissent par le haut et en amont via des processus politiques et parlementaires, voire à travers la jurisprudence, elles bougent aussi sous l'effet du travail de l'administration, c'est-à-dire de ce "processus de création normative continu » (Lascoumes 1990) qu'implique l'application et donc l'interprétation des textes juridiques, conduisant à l'élaboration de " règles secondaires d'application » plus ou moins formalisées. Ainsi, en amont, en parallèle et en conséquence des changements législatifs et jurisprudentiels, on peut observer une production normative par le bas via l'élaboration d'une pratique interne concernant les 'persécutions liées au genre', qui contribue à redéfinir le droit d’asile.

\section{Élaborer une pratique interne : entre évolutions internationales et 'nouveaux motifs'}

Au début des années 1990, avec l'arrivée de nombreuses réfugiées d'ex-Yougoslavie ayant subi de graves violences sexuelles et que l'ODM doit auditionner, les questions de persécutions spécifiques aux femmes deviennent prégnantes, en particulier pour les personnes chargées d'instruire ces dossiers. L’ODM est ainsi amené à faire évoluer sa pratique et à mieux former son personnel, confronté à des situations de plus en plus fréquentes. C'est notamment sous l'impulsion d'une fonctionnaire, psychologue de formation et engagée pour la défense des droits des femmes ou, du moins, sensibilisée à ces questions ${ }^{16}$ que celles-ci sont progressivement prises en compte au sein de l'office.

${ }^{16}$ Cette personne n'est aujourd'hui plus responsable de cette pratique. 
En 1997, à la suite du HCR et des instances européennes, la direction de l'ODR décide de rattacher juridiquement ces persécutions aux raisons liées à "I'appartenance à un groupe social déterminé » (art. 3 al. 1 LAsi). La notion de 'groupe social déterminé' désigne, selon l’ODM :

Un groupe constitué de personnes qui, en raison de certaines qualités innées et immuables, se distingue clairement d'autres groupes de personnes et qui est, du fait de ces qualités, exposé ou craint de l'être à une persécution étatique tolérée par l'État ${ }^{17}$.

En se fondant sur différents textes de référence des organisations internationales, l'ODM reconnaît actuellement sept 'groupes sociaux déterminés' de personnes victimes (i) de mutilations génitales féminines (MGF) ${ }^{18}$, (ii) de violences domestiques, (iii) de mariage forcé, (iv) de crimes d'honneur, (v) de persécutions liées à l' 'orientation sexuelle (LGBT)' ou (vi) à une législation sexiste punissant les atteintes au code moral et enfin (vii) de politiques de l'enfant unique, d'avortement forcé ou de stérilisation forcée ${ }^{19}$. Dans tous ces cas, les fonctionnaires doivent se prononcer sur la 'pertinence' des motifs d'asile, mais aussi sur l'existence d'une protection adéquate dans le pays d'origine, sur la 'vraisemblance' des motifs invoqués (art. 7 LAsi), ainsi que sur la question de l'exigibilité d'un renvoi (art. 44 LAsi) et de l'octroi d'une admission provisoire.

Dans l'examen de la pertinence des motifs liés au genre, ceuxci sont appréciés de manière subsidiaire par rapport aux autres motifs d'asile énumérés à l'article 3 LAsi :

Le motif de persécution découlant de l'appartenance à un groupe social déterminé doit être apprécié comme un élément de fait subsidiaire. En d'autres termes, le motif fondé sur l'appartenance à un groupe social déterminé ne doit être retenu que restrictivement et en l'absence d'autres motifs de persécution ${ }^{20}$.

\footnotetext{
${ }^{17}$ Diaporama diffusé lors d'une formation à l’ODM : « Persécutions liées au genre (Gespever) », notes de terrain, janvier 2012.

${ }^{18}$ À noter que seule la crainte d'une future mutilation et non celle déjà subie est déterminante en matière d'asile.

${ }^{19}$ Pour plus de détails : Barzé 2012 ; Manuel de procédure de l’ODM (2008) «Chapitre J. §2. La problématique des persécutions liées au sexe dans le domaine de l'asile », disponible sur le site de l'ODM : www.bfm.admin.ch ${ }^{20}$ Manuel de procédure de l’ODM (2008), Chap. J. §2 opus cit., p. 6.
} 
L’ODM distingue aussi les persécutions fondées sur l'appartenance à un 'groupe social déterminé' et les 'atteintes d'ordre sexuel' qui peuvent donner droit à l'asile si elles se fondent sur l'un des motifs énoncés au premier alinéa de la loi. Le caractère subsidiaire des 'persécutions liées au genre' et le fait de les rattacher à l'appartenance à 'un groupe social déterminé' font l'objet de critiques. Comme le soulignent plusieurs auteures (Gafner, Schmidlin 2007 ; Stichelbaut 2009), cette manière de faire occulte le caractère politique de ces persécutions, des rapports de genre et de la transgression des règles de comportement genrées.

S'agissant de la terminologie retenue, au cours d'une formation interne, les deux responsables de la pratique, distinguent le genre - 'appartenance sexuelle' qui renvoie aux « relations entre les hommes et les femmes basées sur des identités définies et construites socialement ou culturellement, sur des fonctions, des rôles et des responsabilités qui sont attribués aux hommes et aux femmes (= «gender»)»—- du sexe («identité sexuelle» biologique) ${ }^{21}$. Dans le chapitre du manuel de l'ODM relatif aux 'persécutions liées au sexe' ${ }^{22}$, la distinction entre sexe et genre permet d'inclure tant les 'persécutions spécifiques aux femmes', celles 'spécifiques aux hommes' et celles 'en raison du comportement sexuel (homosexualité)'. Liselotte Barzé, juriste à l’ODM, confirme cette analyse dans un chapitre d'ouvrage qui présente la pratique de l'office en matière de 'persécutions liées au genre'. Elle y explique que le terme de genre permet de comprendre des persécutions qui ne sont pas "infligées en raison $d u$ seul sexe d'une personne ", mais qui "se dirigent principalement à l'encontre de personnes qui refusent de se conformer aux critères sociaux attribués aux hommes et aux femmes [...]. En d'autres termes, le facteur à l'origine du châtiment ou de l'abus n'est pas le sexe biologique de la victime mais la façon dont celle-ci exprime son identité ou son rôle » (Barzé 2012, p. 71).

\footnotetext{
${ }^{21}$ Diaporama, opus cit.

${ }^{22}$ Manuel de procédure de l’ODM (2008), Chap. J. §2 opus cit., p. 4.
} 
Concernant l'orientation sexuelle et l'identité de genre, l’ODM a devancé la jurisprudence européenne ${ }^{23}$, en ayant déjà suivi les recommandations formulées au niveau international, qui considèrent que les personnes qui invoquent des persécutions liées à l'orientation sexuelle et à l'identité de genre peuvent être considérées comme appartenant à 'un groupe social déterminé'. Cette évolution à l'interne illustre aussi la relative perméabilité de l'administration aux 'nouvelles situations' auxquelles elle est confrontée et aux critiques externes dont elle fait l'objet. Ainsi, l'ODM a dû réévaluer sa pratique à l'aune d'une nouvelle question juridique à résoudre. Les responsables de la pratique Gespever ont, en effet, constaté un problème dans l'argumentation et la façon d'apprécier ces motifs d'asile qui ne seraient plus conformes au droit ou, du moins, à l'appréciation actuelle de cette problématique. Cette adaptation de l'interprétation juridique découle aussi de critiques externes qui peuvent émaner des organisations internationales, de la sphère politique ou, plus directement, des milieux associatifs (féministes, collectifs LGBT, organisations de défense du droit d'asile). C'est aussi dans un dialogue avec ces différent·e's actrices et acteurs lors de formations communes que les responsables de la pratique Gespever intègrent des critiques ou de nouvelles problématiques. Cette pratique est donc en constante évolution, notamment à partir des cas concrets que les fonctionnaires doivent traiter, lesquels posent des questions juridiques en particulier sur l'interprétation de l'article 3 LAsi.

\section{Un « continuel combat » à l'interne}

Toutefois, si la pratique peut être perméable à l'évolution des demandes d'asile et à la manière de concevoir les 'persécutions liées au genre' hors de l'ODM, ce n'est pas pour autant que les agent·e·s de l'administration suivent nécessairement ces changements. Par exemple, concernant les problèmes que rencontrent les personnes transsexuelles dans la procédure d'asile, les responsables de la pratique doivent mener tout un travail de sensibilisation à l'interne de l'office pour lutter contre les stéréotypes

${ }^{23}$ Le 7 novembre 2013, un arrêt de la Cour de justice de l’Union européenne a énoncé formellement que les personnes homosexuelles peuvent constituer un groupe social. 
ou les clichés de certain·e·s collègues ${ }^{24}$. Cette sensibilisation peut paraître d'autant plus compliquée que la participation aux formations sur ces questions n'est pas forcément obligatoire et que la majorité des personnes qui y participent sont des femmes. Or, l'Ordonnance sur l'asile OA1 prévoyant l'audition "par des personnes du même sexe » lorsqu'il y a des indices de persécutions de "nature sexuelle ", les hommes peuvent aussi être amenés à auditionner des hommes invoquant des 'persécutions liées au genre'. Ainsi, ces formations (notamment sur les problématiques 'liées aux personnes LGBT') ont été progressivement imposées aux collaboratrices et collaborateurs de l'office.

Si certain·e·s collègues peuvent se montrer réticent $\cdot e \cdot s$ ou, du moins, peu intéressé·e·s, la sensibilisation aux 'persécutions liées au genre' doit aussi passer par un "continuel combat» auprès des différents échelons de la hiérarchie de l'ODM pour faire reconnaître l'importance de développer et de respecter cette pratique. Dans un contexte marqué par une volonté d'accélérer le traitement des demandes d'asile et d'en réduire le nombre, la faible proportion des demandes d'asile invoquant des 'persécutions liées au genre' ${ }^{25}$ ne figure pas vraiment parmi les priorités de traitement et d'allocation des ressources. Le « continuel combat» interne est d'autant plus difficile qu'il n'existe aucun moyen vraiment contraignant pour contrôler le respect de la pratique et qu'elles sont soumises à différentes contraintes institutionnelles - par exemple, les évolutions majeures de la pratique doivent être discutées et avalisées par la hiérarchie.

\footnotetext{
${ }^{24}$ Selon les observations et entretiens réalisés au sein de l’ODM.

${ }^{25}$ Selon des statistiques internes de l’ODM, en 2012, les décisions sur des 'persécutions liées au genre' représentaient au moins $5 \%$ du total des décisions d'asile et $8 \%$ des décisions d'octroi de l'asile, $11 \%$ des rejets (avec et sans admission provisoire), $3 \%$ des décisions de non-entrée en matière et 2 \% des radiations. Pour 2012, 1351 décisions ont été rendues concernant des demandes invoquant des 'persécutions liées au genre'. Parmi celles-ci, 191 $(14,1 \%)$ ont obtenu la qualité de réfugié·e, alors que le taux d’octroi de l'asile pour l'ensemble des décisions rendues en 2012 était de 11,7 \%. Enfin, pour les décisions négatives et de non-entrée en matière, 130 admissions provisoires ont été prononcées (9,6 \%), contre un taux de 7,4\% pour l'ensemble des décisions.
} 
Depuis l'instauration d'une pratique en matière de 'persécutions liées au genre', les personnes responsables ont toujours été uniquement des femmes. Les responsables actuelles de la pratique Gespever - toutes deux ont suivi un cursus universitaire en droit - disent ne pas avoir eu d'engagements militants avant de travailler à l'ODM. Elles ont dû se former à ces questions 'sur le tas', en lisant des rapports, en participant à des formations en Suisse et à l'étranger, en dialoguant avec des associations et, surtout, en étant confrontées à de nouveaux cas de figure. C'est en traitant 'des cas comme ça', en s'intéressant à ces thématiques et "en sortant des cadres de l'ODM » via la participation à des formations et autres manifestations qu'elles ont été sensibilisées à ces questions. Ainsi, elles sont amenées à dialoguer, hors de l'ODM, avec différent·e·s actrices et acteurs associatifs ou avec des expert·e·s. Leur travail à l'interne (développement de la pratique, sensibilisation) peut être analysé comme une forme de continuité « intra-institutionnelle » (Bereni, Revillard 2012) des mobilisations féministes, puis LGBT, qui ont porté les questions de 'persécutions liées au genre'. On peut analyser le «continuel combat » auquel il a été fait référence comme une forme de relais de ces mobilisations à l'interne de l'administration afin de faire évoluer le droit et son interprétation. Pour autant, les responsables de la pratique Gespever ne se perçoivent pas comme telles, et encore moins comme des militantes - étiquette dont elles cherchent justement à se distancier. À cet égard, elles ont travaillé à rompre avec une approche perçue comme "militante" pour développer une « approche juridique exempte de subjectivité, permettant de mener des débats sur le fond dénués de toute émotivité $"{ }^{26}$. Cette dépolitisation par le droit contribue à donner davantage de légitimité à cette pratique auprès des collègues et de la hiérarchie de l'ODM.

Cette situation peut être génératrice de tensions. Si, en développant la pratique Gespever, ces femmes sont susceptibles de relayer, en partie, des revendications portées par différentes mobilisations collectives, elles n'en demeurent pas moins les employées d'un office ayant ses logiques propres et dont le

${ }^{26}$ Échange écrit avec les responsables de la pratique Gespever. 
travail de contrôle des demandes d'asile se caractérise par une logique de suspicion - imprégnée de la thématisation de l'asile en termes "d'abus" et de "faux réfugiés " (Maillard, Tafelmacher 1999) — et de productivité, liée à une forme de « gestion du stock » des requérant·e·s d'asile (Tafelmacher 2013). C'est dire que l'élaboration d'une pratique Gespever s'accompagne aussi d'un travail visant à trouver une «bonne manière » d'instruire les dossiers, d'auditionner les personnes invoquant des motifs de persécution liés au genre et, partant, de juger la "pertinence » (art. 3) et la "vraisemblance » (art. 7 LAsi) des histoires racontées par les requérant·e·s d'asile en audition. Comme l'a montré un rapport de l'ONG Terre des femmes, ce contrôle est le principal obstacle à l'acceptation des demandes d'asile de femmes, en cela qu'il amène à discréditer leur témoignage ${ }^{27}$. Cette tension ressort clairement des entretiens menés au sein de l'ODM. Invoquant le cadre législatif qui les oblige à apprécier les déclarations d'une personne également en relation avec un événement traumatisant et différenciant clairement leur travail de celui des psychiatres (experts des traumatismes) ou des policiers (cherchant la vérité), les agent·e·s de l'ODM sont pris·e·s dans un cadre légal qui les incite à «ne pas croire tout le monde » et à "faire au mieux dans le cadre qui leur a été donné pour arriver à un résultat ${ }^{28}$, autrement dit parvenir à une décision 'acceptable' juridiquement et institutionnellement.

L'évaluation de la crédibilité de motifs d'asile amène les fonctionnaires à questionner les requérant·e·s d'asile sur les violences subies, notamment le(s) viol(s) subi(s) («Racontez-moi ce viol ${ }^{29}$ ), ou sur l'homosexualité elle-même, pour prendre un exemple déjà étudié (Morgan 2006 ; Kobelinsky 2012). L’enjeu de l'audition devient alors de 'rendre crédibles' les violences

\footnotetext{
${ }^{27}$ Wiebke Doering (2011). « Les femmes dans la procédure d’asile. La reconnaissance des motifs de fuite spécifiques aux femmes dans la pratique de l'asile en Suisse ». Terre des Femmes, décembre 2011, disponible sur le site : www.terre-des-femmes.ch

${ }^{28}$ Selon des entretiens réalisés au sein de l’ODM.

${ }^{29}$ Pour un exemple extrême : ODAE. « L'attitude choquante des autorités lors de l'audition d'une femme violée ». Infos brèves, 15.02.2010, disponible sur le site http://odae-romand.ch/spip.php?article217
} 
sexuelles subies et de montrer que l'on est "assez gay" (Morgan 2006) pour obtenir l'asile.

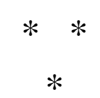

La question du genre s'est posée au droit d'asile par le biais des 'persécutions liées au genre'. Cette problématique a été portée par les organisations internationales, des mobilisations et des interventions dans la sphère politique nationale qui ont abouti à une révision législative visant à tenir compte des 'persécutions liées au genre'. Une analyse de la production du droit en la matière ne saurait toutefois faire l'économie du rôle considérable joué par l'administration. Celle-ci se saisit, en effet, de cette question tant en amont, en parallèle - sous l'effet de situations 'nouvelles' invoquées dans les demandes d'asile, et de critiques externes - , qu'en conséquence des débats internationaux et nationaux. La pratique développée par l'ODM privilégie une 'solution' juridique (inspirée par le HCR) consistant à rattacher différentes situations de 'persécutions liées au genre' à l'une des raisons donnant droit à l'asile et figurant à l'article 3 LAsi, à savoir l'appartenance à un 'groupe social déterminé'. À mesure que de nouvelles problématiques se posent, la pratique continue à évoluer, sous l'effet aussi de celles qui en ont la charge et qui travaillent à l'interne à la développer et à trouver des solutions juridiques qui puissent aider et guider leurs collègues pour l'instruction des dossiers et pour la décision.

On peut analyser ce travail comme une forme de relais « intra-institutionnel » (Bereni, Revillard 2012) de revendications portées par différents acteurs internationaux, politiques et associatifs. Cette dimension de relais doit toutefois être relativisée au regard de différentes contraintes institutionnelles qui pèsent sur le travail de ces agentes. Elles sont notamment prises dans une tension entre, d'une part, une logique d'ouverture relative à l'égard d'une perspective visant à prendre en compte la dimension genrée des persécutions et, d'autre part, des logiques institutionnelles de suspicion et de "gestion du stock» (Tafelmacher 2013) qui imprègnent le travail de contrôle des demandes d'asile. On le voit, l'administration participe à la pro- 
duction du droit en se réappropriant et en réinterprétant la notion de genre selon des logiques qui lui sont propres. Le passage par le droit et le développement d'une approche juridique des 'persécutions liées au genre' contribuent ainsi à mettre à distance la dimension militante des revendications portées notamment par les mobilisations féministes et LGBT, à les dépolitiser, voire à les neutraliser sous les auspices d'une catégorisation juridique tendant à euphémiser le potentiel critique de la notion de genre.

Ainsi, s'il a été progressivement possible d'accorder l'asile à des personnes victimes de violences de tiers et notamment de violences sexuelles, et si les 'persécutions liées au genre' ont été en partie intégrées dans les textes juridiques et dans la pratique de l'office, cette prise en compte est ambivalente. D'un côté, les textes juridiques et la pratique de l'ODM cherchent à inclure la dimension genrée des persécutions et à corriger 'à reculons' la définition des réfugié·e•s. D’un autre côté, ce développement ne se fait qu'à moitié puisque les 'persécutions liées au genre' ne sont pas, en elles-mêmes, des motifs d'asile au même titre que ceux qui figurent à l'art. 3 al. 1 LAsi. Elles apparaissent donc comme 'secondaires' dans la mesure où ces derniers priment sur elles. Par ailleurs, comme l'ont déjà souligné d'autres articles (Gafner 2003 ; Stichelbaut 2009), privilégier la notion de 'groupe social déterminé' contribue à écarter la dimension politique du genre en tant que rapport social.

\section{Références}

Barzé Liselotte (2012). «La pratique de l’Office fédéral des migrations (ODM) en matière de persécutions liées au genre ». In Achermann Alberto, Hruschka Constantin (eds). Persécutions liées au genre. La pratique suisse au regard des évolutions européennes et globales. Berne, Weblaw.

Bereni Laure, Chauvin Sébastien, Jaunait Alexandre, Revillard Anne (2012). Introduction aux études sur le genre. Bruxelles, de Boeck [2 éd.].

Bereni Laure, Debauche Alice, Latour Emmanuelle, Lempen Karine, Revillard Anne (eds) (2009). " Le droit à l'épreuve du genre : les lois du genre (I) ». Nouvelles questions féministes, vol. 28, nº 2. 
Bereni Laure, Debauche Alice, Latour Emmanuelle, Revillard Anne (eds) (2010). «Quand les mouvements féministes font (avec) la loi : les lois du genre (II) ». Nouvelles questions féministes, vol. 29, $\mathrm{n}^{\circ} 1$.

Bereni Laure, Revillard Anne (2012). " Un mouvement social paradigmatique ? Ce que le mouvement des femmes fait à la sociologie des mouvements sociaux ». Sociétés contemporaines, $\mathrm{n}^{\circ} 85$.

Bertschi Martin (2007). " Droits des étrangers et droit d'asile ». In Ziegler Andreas R. et al. Droits des gays et lesbiennes en Suisse. Partenariat enregistré, communauté de vie de fait, questions juridiques concernant l'homosexualité. Berne, Stämpfli.

Bhabha Jacqueline (1996). "Embodied Rights: Gender Persecution, State Sovereignty and Refugees". Public Culture, vol. 9, $\mathrm{n}^{\circ} 1$.

Bohmer Carol, Shuman Amy (2008). Rejecting Refugees. Political Asylum in the 21st Century. London \& New York, Routledge.

Chevallier Jacques (2011). « La place de l'administration dans la production des normes ». Droit et société, $\mathrm{n}^{\circ} 79$.

Freedman Jane (2008). «Genre et migration forcée: les femmes exilées en Europe ». Les Cahiers du CEDREF, $\mathrm{n}^{\circ} 16$.

- (2010a). " Les mobilisations féministes autour du droit d'asile en France et au Royaume-Uni : des normes internationales au droit national ». Nouvelles questions féministes, vol. 29, nº 1.

- (2010b). "Mainstreaming Gender in Refugee Protection". Cambridge Review of International Affairs, vol. 23, $n^{\circ} 4$.

Gafner Magalie (2003). « Les femmes migrantes face à la loi sur le séjour et l'établissement des étrangers (LSEE), à la loi sur les étrangers (LEtr) et à la loi sur l'asile (LAsi) ». Revue de droit administratif et de droit fiscal, $\mathrm{n}^{\circ} 16$.

Gafner Magalie, Schmidlin Irène (2007). « Le genre et la législation suisse en matière de migration ». Nouvelles questions féministes, vol. 26, $n^{\circ} 1$.

Hausammann Christina (1992). Les femmes victimes de persécutions et la notion de réfugié. Comment interpréter le terme de "réfugié » figurant dans la Convention relative au statut des réfugiés et dans la loi sur l'asile ? Berne, Bureau fédéral de l'égalité entre femmes et hommes.

Jenson Jane, Lépinard Éléonore (2009). " Penser le genre en science politique. Vers une typologie des usages du concept». Revue française de science politique, vol. 59, $\mathrm{n}^{\circ} 2$.

Kobelinsky Carolina (2012). «L'asile gay : jurisprudence de l'intime à la cour nationale du droit d'asile ». Droit et société, nº 82. 
Lascoumes Pierre (1990). « Normes juridiques et mise en œuvre des politiques publiques ». L'Année sociologique, vol. 40.

Lépinard Éléonore (2006). « Faire la loi, faire le genre : conflits d'interprétations juridiques sur la parité ». Droit et société, n 62.

Maillard Alain, Tafelmacher Christophe (1999). "Faux réfugiés »? La politique suisse de dissuasion d'asile, 1979-1999. Lausanne, Éd. d'en bas.

Morgan Deborah A. (2006). "Not Gay Enough for the Government: Racial and Sexual Stereotypes in Sexual Orientation Asylum Cases". Law \& Sexuality Review. Lesbian Gay Bisexual \& Legal Issues, $n^{\circ} 15$.

Stichelbaut Françoise (2009). "L'application de la Convention de l'ONU sur les réfugiés aux demandeuses d'asile lesbiennes : de quel genre parlons-nous ? » Nouvelles questions féministes, vol. 28, $\mathrm{n}^{\circ} 2$.

Tafelmacher Christophe (2013). « Du droit d'asile à la gestion du stock humain ou comment réduire à néant l'hospitalité et les droits ». Vivre ensemble, hors-série, $n^{\circ} 3$.

\section{Liste des sigles utilisés}

CRA - Commission suisse de recours en matière d'asile

HCR - Haut commissariat des Nations unies pour les réfugiés

JICRA - Jurisprudence et informations de la CRA

LGBT - Lesbiennes, Gays, Bi, Trans

LAsi - Loi sur l'asile

MGF - Mutilations génitales féminines

IMES - Office fédéral de l'immigration, de l'intégration et de l'émigration

ODM - Office fédéral des migrations

ODR - Office fédéral des réfugiés

OA - Ordonnance d'application de la loi sur l'asile

ONG - Organisation non gouvernementale

Gespever - Geschlechtsspezifische Verfolgung (Persécutions liées au genre)

TAF - Tribunal administratif fédéral 